\title{
Pacific
}

Journal of

Mathematics

\section{SEMIQUANDLES AND FLAT VIRTUAL KNOTS}

ALlison HenRICH AND SAM NELSON 


\title{
SEMIQUANDLES AND FLAT VIRTUAL KNOTS
}

\author{
ALlison HenRich AND SAM NELSON
}

\begin{abstract}
We define an algebraic structure we call a semiquandle, whose axioms are derived from the flat Reidemeister moves. Finite semiquandles have associated counting invariants and enhanced invariants, defined for flat virtual knots and links. We also introduce singular semiquandles and virtual singular semiquandles, which define invariants of flat singular virtual knots and links. As an application, we use semiquandle invariants to compare two Vassiliev invariants.
\end{abstract}

\section{Introduction}

Recent works, such as [Kauffman 1999], take a combinatorial approach to knot theory, in which knots and links are regarded as equivalence classes of knot and link diagrams. New types of combinatorial knots and links can then be defined by introducing new types of crossings and Reidemeister-style moves that govern their interactions. These new combinatorial classes of knots and links have various topological and geometric interpretations relating to simple closed curves in 3manifolds, rigid vertex isotopy of graphs, and so on.

A flat crossing is a classical crossing in which we ignore the over/under information. A flat knot or link is a planar projection (or shadow) of a knot or link, on the surface on which the knot or link diagram is drawn. Every classical knot diagram may be regarded as a lift of a flat knot and, conversely, every classical knot diagram has a corresponding flat shadow.

Flat knots might seem uninteresting since flattening classical crossings apparently throws away the information which defines the knotting. However, a little thought reveals potential applications of flat crossings; for example, invariants of links with classical intercomponent crossings and flat intracomponent crossings are related to link homotopy and the Milnor invariants of ordinary classical links.

Flat crossings also prove useful in virtual knot theory. Every purely flat knot is trivial, that is, reducible by flat Reidemeister moves to the unknot. However, flat virtual knots and links (that is, diagrams with virtual and flat crossings) are

MSC2000: 57M25, 57M27.

Keywords: flat knots and links, virtual knots and links, singular knots and links, semiquandles,

Vassiliev invariants. 
generally nontrivial. The nontriviality of a flat virtual knot says that no choice of classical crossing information for the flat crossings would yield a classical knot. Hence, flat crossings are useful in the study of nonclassicality for virtual knots.

A singular crossing is a crossing where two strands are fused together. Singular knots and links may be understood as rigid vertex isotopy classes of knotted and linked graphs, and they play a role in the study of Vassiliev invariants of classical knots and links.

In this paper, we define an algebraic structure we call a semiquandle, which yields counting invariants for flat virtual knots. The paper is organized as follows. In Section 2, we define flat, singular, and virtual knots and links. In Section 3, we define semiquandles and give some examples. In Section 4, we define singular semiquandles by including operations at singular crossings. In Section 5, we introduce virtual semiquandles and virtual singular semiquandles by including an operation at virtual crossings. In Section 6, we give examples to show that the counting invariants with respect to finite semiquandles can distinguish flat virtual knots and links. In Section 7, we give an application to Vassiliev invariants of virtual knots. In Section 8, we collect some questions for further research.

\section{Flat knots, virtual knots and singular knots}

We introduce several types of knots discussed in this paper. We assume that all knots are oriented, unless otherwise specified. The simplest type of knot among those we consider, a flat knot, is an immersion of $S^{1}$ in $\mathbb{R}^{2}$. Alternatively, a flat knot can be described as an equivalence class of knot diagrams where under/over strand information at each crossing is unspecified. Their equivalence relation is given by flat versions of the Reidemeister moves, illustrated here:
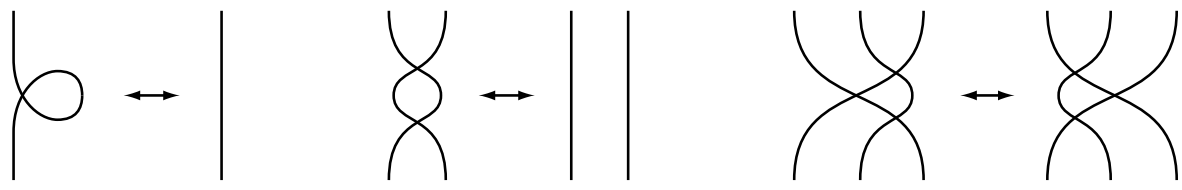

It is an easy exercise to show that any flat knot is related by a sequence of flat Reidemeister moves to the trivial flat knot (that is, the flat knot with no crossings). While the theory of flat knots appears uninteresting, if we consider the analogous theory of flat virtual knots, we enter a highly nontrivial category.

A flat virtual knot is a decorated immersion of $S^{1}$ in $\mathbb{R}^{2}$, where each crossing is decorated to indicate whether it is flat or virtual. (Virtual crossings are pictured by an encircled flat crossing.) Once again, we may also describe a flat virtual knot as an equivalence class of virtual knot diagrams where under/over strand information at each classical crossing is unspecified. The corresponding equivalence relation is given by the flat versions of the virtual Reidemeister moves, in addition to the 
flat versions of the ordinary Reidemeister moves.
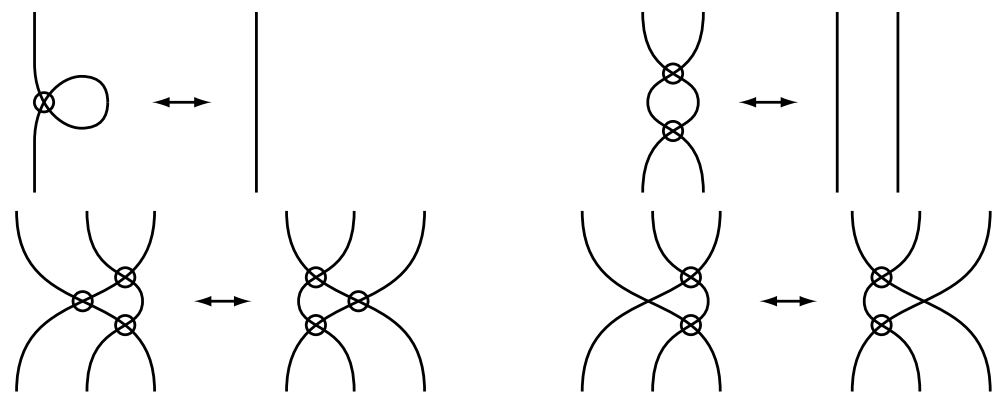

The move
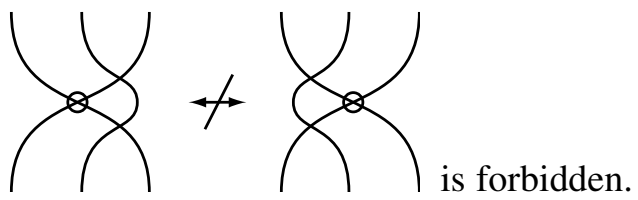

As with ordinary virtual knots, flat virtual knot diagrams have a geometric interpretation as flat knot diagrams on surfaces. In this case, the virtual crossings are interpreted as artifacts of a projection of the knot diagram on the surface to a knot diagram in the plane [Kauffman 1999].

Finally, we consider flat knots and flat virtual knots that have singularities. The singularities should be thought of as rigid vertices, or places where the knot is actually glued to itself. Thus, flat singular knots are simply equivalence classes of flat knots where some crossings are decorated to indicate that they are singular. The Reidemeister moves corresponding to flat singular equivalence are the ordinary flat equivalence moves together with these two moves:
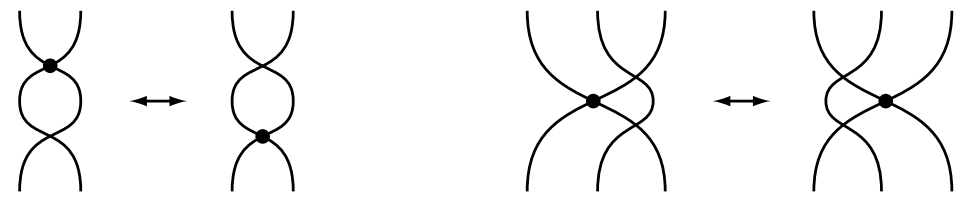

Similarly, flat virtual singular knots are equivalence classes of flat virtual knots where some of the crossings may be designated as singular. Hence, there are three types of crossings that may be contained in a diagram of a flat virtual singular knot. The equivalence relation is given by all of the previous flat, virtual, and singular moves, together with this:

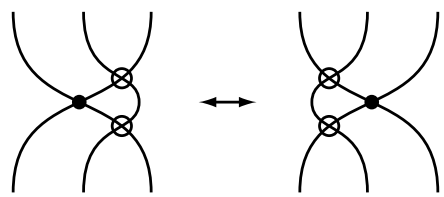


The simplest nontrivial flat virtual singular knot that contains all three types of crossings is the triple trefoil:

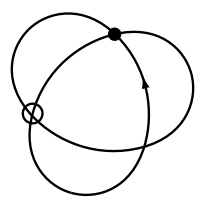

\section{Semiquandles}

In recent years, a number of algebraic structures have been defined with axioms derived from variations on the oriented Reidemeister moves. The earliest of these is the quandle [Joyce 1982; Matveev 1982], in which we have generators corresponding to arcs in a link diagram, and an invertible binary operation at crossings.

Subsequent papers have generalized this idea in various ways. Fenn and Rourke [1992] replace ambient isotopy with framed isotopy to define racks. In [Kauffman and Radford 2003; Fenn et al. 1995], arcs in an oriented knot diagram are replaced with semiarcs, to define biquandles. Kauffman and Manturov [2005] include an operation at virtual crossings in the biquandle definition to yield virtual biquandles.

Definition 1. A semiquandle is a set $X$ with two binary operations $(x, y) \mapsto x^{y}$ and $(x, y) \mapsto x_{y}$ such that, for all $x, y, z \in X$,

(0) there are unique $w$ and $z \in X$ with $x=w^{y}$ and $x=z_{y}$;

(i) $x_{y}=y$ if and only if $y^{x}=x$;

(ii) $\left(x_{y}\right)^{\left(y^{x}\right)}=x$ and $\left(x^{y}\right)_{\left(y_{x}\right)}=x$;

(iii) $\left(x^{y}\right)^{z}=\left(x^{z_{y}}\right)^{y^{z}},\left(y_{x}\right)^{z_{x} y}=\left(y^{z}\right)_{x^{z y}}$ and $\left(z_{x}\right)_{y_{x}}=\left(z_{y}\right)_{x}$.

Axiom (0) says that the actions $x \mapsto x^{y}$ and $x \mapsto x_{y}$ are invertible; these unique $z$ and $w$ will be denoted by $z=x_{y^{-1}}$ and $w=x^{y^{-1}}$. The axioms come from dividing an oriented flat knot into semiarcs, that is, edges between vertices in the flat diagram regarded as a graph, and then translating the flat Reidemeister moves into algebraic axioms.

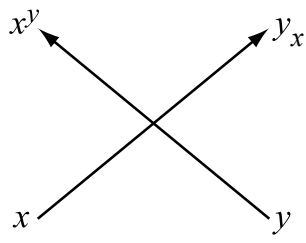

In the first Reidemeister move, right invertibility guarantees the uniqueness of $y$ given $x$, and the relationship between $x$ and $y$ becomes axiom (i). 


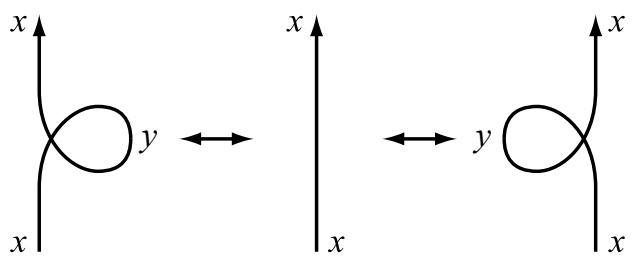

The direct II move, in which both strands are oriented in the same direction, gives us axiom (ii). Given axiom (0), the reverse II move yields the same relationship between $x$ and $y$, where the uncrossed strands are labeled by $x$ and $y^{x}$.
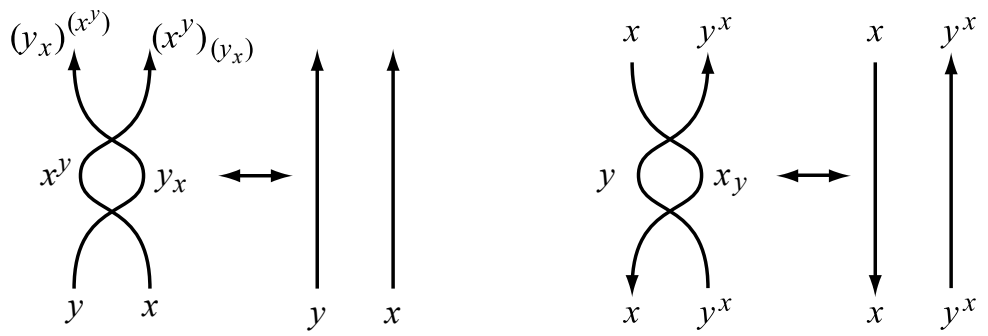

Reidemeister move III yields the three equations in axiom (iii).

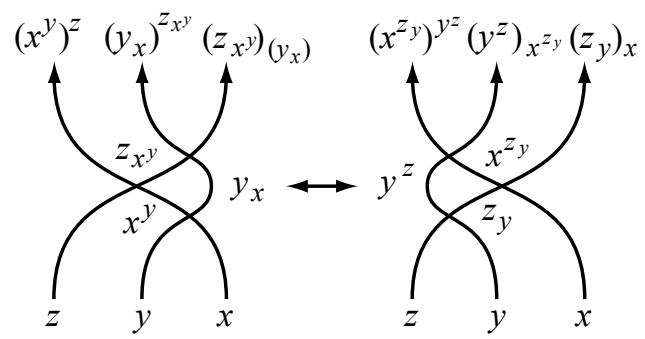

Definition 2. For any flat virtual link $L$, the fundamental semiquandle $\operatorname{FSQ}(L)$ of $L$ is the set of equivalence classes of semiquandle words in a set of generators corresponding to semiarcs in a diagram $D$ of $L$, that is, edges in the graph obtained from $D$ by considering flat crossings as vertices under the equivalence relation generated by the semiquandle axioms and the relations at the crossings. As with the knot quandle, fundamental rack, and knot biquandle, we can express the fundamental semiquandle with a presentation read from a diagram.

Example 3. The flat Kishino knot is

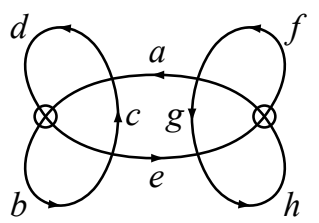


and has the fundamental semiquandle presentation

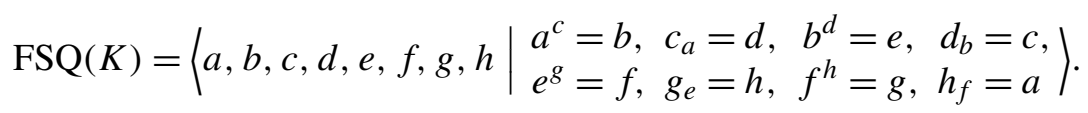

Remark 4. An alternative definition for the fundamental semiquandle of a flat virtual knot is that $\operatorname{FSQ}(L)$ is the quotient of the (strong) knot biquandle of any lift of $L$ (that is, of any choice of classical crossing type for the flat crossings of $L$ ), under the equivalence relation generated by setting $a^{\bar{b}} \sim a_{b}$ and $a_{\bar{b}} \sim a^{b}$ for all $a, b \in B(L)$. Indeed, this operation yields a "flattening" functor SQ : $\mathscr{B} \rightarrow \mathscr{Y}$ from the category of strong biquandles to the category of semiquandles.

Example 5. For any set $X$ and bijection $\sigma: X \rightarrow X$, the operations $x^{y}=\sigma(x)$ and $x_{y}=\sigma^{-1}(x)$ define a semiquandle structure on $X$. We call this type of semiquandle a constant-action semiquandle, since the action of $y$ on $x$ is constant as $y$ varies.

As is the case with quandles and biquandles [Ho and Nelson 2005; Nelson and Vo 2006], for a finite semiquandle $X=\left\{x_{1}, \ldots, x_{n}\right\}$ we can conveniently express the semiquandle structure with a block matrix $M_{X}=[U \mid L]$, where $U_{i, j}=k$ and $L_{i, j}=l$ for $x_{k}=\left(x_{i}\right)^{\left(x_{j}\right)}$ and $x_{l}=\left(x_{i}\right)_{\left(x_{j}\right)}$. This matrix notation enables us to do computations with semiquandles without the need for formulas for $x^{y}$ and $x_{y}$.

Example 6. The constant-action semiquandle on $X=\{1,2,3\}$ with $\sigma=(132)$ has semiquandle matrix

$$
M_{X}=\left[\begin{array}{lll|lll}
3 & 3 & 3 & 2 & 2 & 2 \\
1 & 1 & 1 & 3 & 3 & 3 \\
2 & 2 & 2 & 1 & 1 & 1
\end{array}\right]
$$

Example 7. Any (strong) biquandle in which $a^{\bar{b}}=a_{b}$ and $a_{\bar{b}}=a^{b}$ is a semiquandle. Indeed, an alternative name for semiquandles might be symmetric biquandles. An example of a nonconstant action semiquandle, found in [Nelson and Vo 2006], is

$$
M_{T}=\left[\begin{array}{llll|llll}
1 & 4 & 2 & 3 & 1 & 3 & 4 & 2 \\
2 & 3 & 1 & 4 & 3 & 1 & 2 & 4 \\
4 & 1 & 3 & 2 & 2 & 4 & 3 & 1 \\
3 & 2 & 4 & 1 & 4 & 2 & 1 & 3
\end{array}\right] .
$$

\section{Singular semiquandles}

We now consider what happens to our algebraic structure when we allow singular crossings in an oriented flat virtual knot. As with flat crossings, we define two binary operations at a singular crossing. One notable difference is that, unlike flat crossings, singular crossings are permanent - there are no moves that either introduce or remove singular crossings. Indeed, the number of singular crossings 
is an invariant of the singular knot type. In particular, at singular crossings, we do not need right invertibility for our operations.

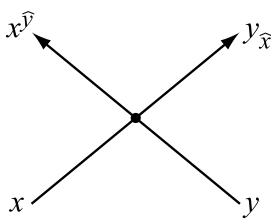

Definition 8. Let $X$ be a semiquandle. A singular semiquandle structure on $X$ is a pair $(x, y) \mapsto x^{\hat{y}}$ and $(x, y) \mapsto x_{\hat{y}}$ of binary operations on $X$ that satisfy, for all $x, y, z \in X$,

(hi) $\left(y_{x}\right)^{\left(\widehat{x^{y}}\right)}=\left(y_{\hat{x}}\right)^{\left(x^{\hat{y}}\right)}$ and $\left(x^{y}\right)_{\left(\widehat{y_{x}}\right)}=\left(x^{\hat{y}}\right)_{\left(y_{\hat{x}}\right)}$;

(hii) $\left(x^{y}\right)^{\hat{z}}=\left(x^{\widehat{z y}}\right)^{y^{z}},\left(y_{x}\right)^{z} \hat{x}=\left(y^{z}\right)_{x^{\widehat{y}}}$, and $\left(z_{x^{y}}\right)_{y_{x}}=\left(z_{y}\right)_{\hat{x}}$.

We call axioms (hi) and (hii) the hat axioms. These axioms come from the subset of the oriented singular flat Reidemeister moves pictured here:
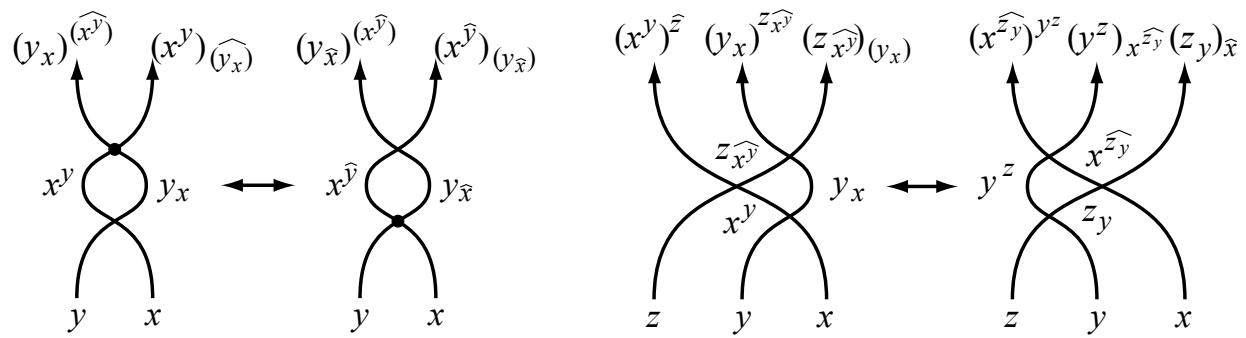

To see that the two pictured oriented singular moves are sufficient to give us all of the oriented flat singular moves, we note the following key lemmas.

Lemma 9. The move

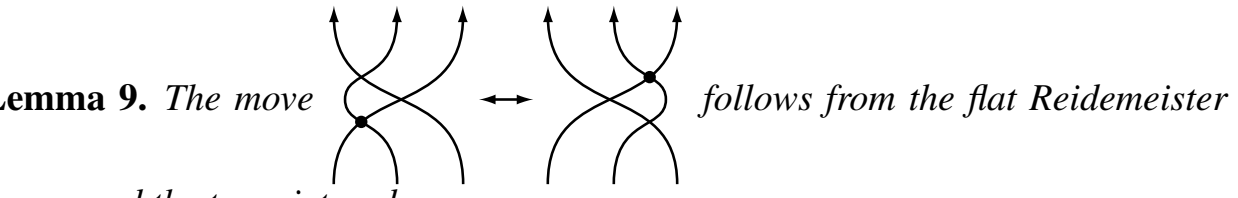

follows from the flat Reidemeister moves and the two pictured moves.

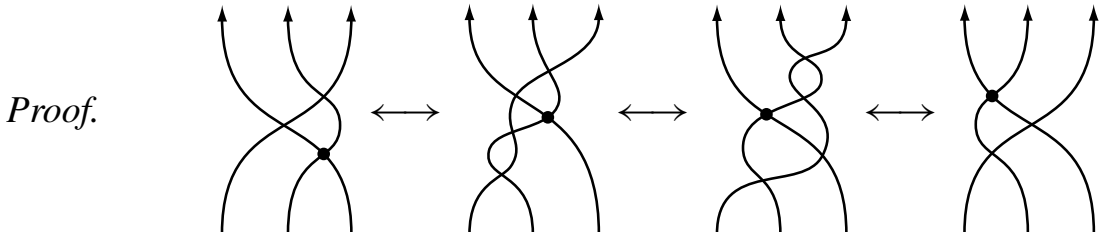

Similar move sequences yield the other oriented flat/singular type III moves.

Lemma 10. The reverse oriented singular II move follows from the flat moves and the moves pictured above. 
Proof. Starting with one side of a reverse singular II move, we can use flat moves to get a symmetric diagram in which we can apply a direct singular II move.

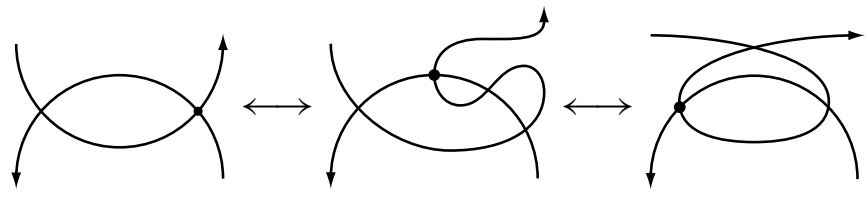

Reversing the process gives the other side of the reverse singular II move.

Example 11. Let $X$ be a semiquandle. Clearly, setting $x^{\hat{y}}=x^{y}$ and $x_{\hat{y}}=x_{y}$ for all $x, y \in X$ defines a compatible singular structure $(X, X)$, which we call the flat singular structure.

Example 12. If $X$ is a semiquandle, then $a^{\hat{b}}=a_{\hat{b}}=b$ is a compatible singular structure, since we have

$$
\begin{array}{ll}
\left(y_{x}\right)^{\left(\widehat{x^{y}}\right)}=x^{y}=\left(y_{\hat{x}}\right)^{\left(x^{\hat{y}}\right)}, & \left(y_{x}\right)^{z \widehat{x y}}=\left(y_{x}\right)^{x^{y}}=y=\left(y^{z}\right)_{\widehat{z_{y}}}=\left(y^{z}\right)_{x^{\widehat{z y}}}, \\
\left(x^{y}\right)_{\left(\widehat{y_{x}}\right)}=y_{x}=\left(x^{\hat{y}}\right)_{\left(y_{\hat{x}}\right)}, & \left(x^{y}\right)^{\hat{z}}=z=\left(z_{y}\right)^{y^{z}}=\left(x^{\widehat{z y}}\right)^{y^{z}}, \\
\left(z_{\widehat{x}}\right)_{y_{x}}=\left(x^{y}\right)_{y_{x}}=x=\left(z_{y}\right)_{\hat{x}} . &
\end{array}
$$

We call this singular structure the operator singular structure on $X$ and denote it by $(X, O)$.

As with the flat virtual case, for any flat singular virtual link $L$ there is an associated fundamental singular semiquandle $\operatorname{FSSQ}(L)$, with presentation readable from the diagram. It elements are equivalence classes of singular semiquandle words, in generators corresponding to semiarcs in the diagram (here, we divide the diagram at both flat and singular crossing points, but not at virtual crossings) under the equivalence relation generated by the axioms (0), (i), (ii), (iii), (hi), and (hii).

Example 13. The triple trefoil below has the fundamental singular semiquandle presentation $\left\langle a, b, c, d \mid a^{\hat{c}}=b, c_{\hat{a}}=d, d^{b}=a, b_{d}=c\right\rangle$.

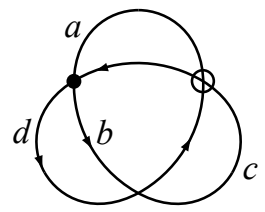

As with the semiquandle structure, we can represent the singular operations in a finite singular semiquandle by matrices encoding the operation tables. Indeed, it seems convenient to combine these matrices with the semiquandle operation 
matrices, into a single block matrix of the form

$$
M_{T}=\left[\begin{array}{c|c}
i^{j} & i_{j} \\
\hline i^{\hat{j}} & i_{\hat{j}}
\end{array}\right] .
$$

Example 14. The constant action semiquandle $X=\{1,2,3\}$ with $\sigma=(132)$ and operator singular structure $a^{\hat{b}}=a_{\hat{b}}=b$ has block matrix

$$
M_{(X, O)}=\left[\begin{array}{lll|lll}
3 & 3 & 3 & 2 & 2 & 2 \\
1 & 1 & 1 & 3 & 3 & 3 \\
2 & 2 & 2 & 1 & 1 & 1 \\
\hline 1 & 2 & 3 & 1 & 2 & 3 \\
1 & 2 & 3 & 1 & 2 & 3 \\
1 & 2 & 3 & 1 & 2 & 3
\end{array}\right] .
$$

\section{Virtual semiquandles and virtual singular semiquandles}

As with singular crossings, we can further generalize semiquandles by adding an operation at virtual crossings. The simplest way to do this is to use a unary operation at each virtual crossing, defined by applying a bijection $v$ when going through a virtual crossing from right to left (looking in the direction of the strand being crossed) and applying $v^{-1}$ when going through a virtual crossing from left to right (looking in the direction of the strand being crossed).

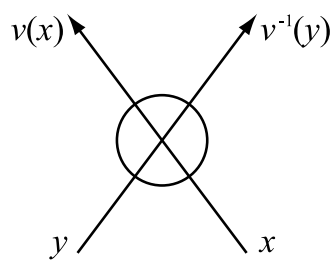

As noted in [Kauffman and Manturov 2005], this setup ensures that the virtual I, II and III moves are respected by the virtual operation.
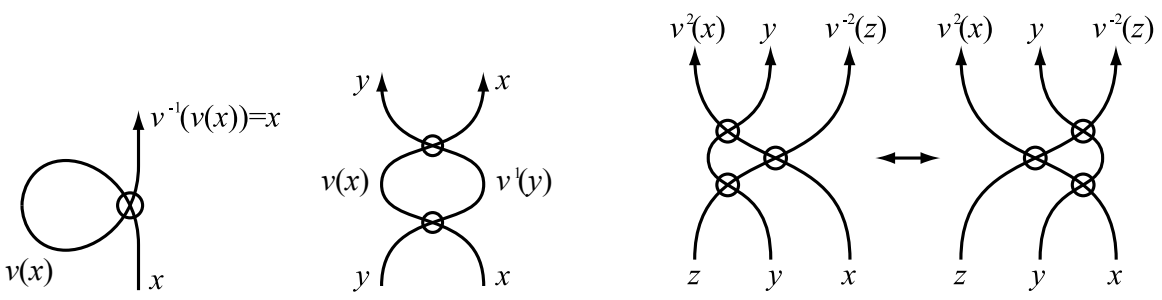

The interaction of the virtual crossings with the flat and singular crossings given by the Reidemeister moves tells us how the virtual operation should interact with 
the semiquandle and singular semiquandle structures; namely, $v$ must be an automorphism of both structures.
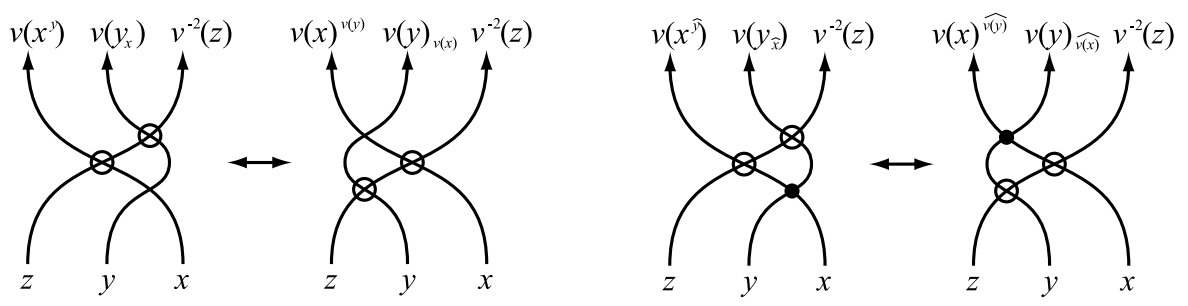

Definition 15. A virtual semiquandle is a semiquandle $S$ with a choice of automorphism $v: S \rightarrow S$. A virtual singular semiquandle is a singular semiquandle with a semiquandle automorphism $v: S \rightarrow S$ that is also an automorphism of the singular structure. That is, $v: S \rightarrow S$ is a bijection satisfying

$$
v\left(x^{y}\right)=v(x)^{v(y)}, \quad v\left(x_{y}\right)=v(x)_{v(y)}, \quad v\left(x^{\hat{y}}\right)=v(x)^{\widehat{v(y)}}, \quad v\left(x_{\hat{y}}\right)=v(x)_{\widehat{v(y)}} .
$$

Example 16. Every semiquandle is a virtual semiquandle with $v=\operatorname{Id}_{S}$. More generally, the set of virtual semiquandle structures on a semiquandle $S$ corresponds to the set of conjugacy classes in the automorphism group $\operatorname{Aut}(S)$ of the semiquandle $S$ : if $v, v^{\prime}, \varphi \in \operatorname{Aut}(S)$ with $v^{\prime}=\varphi^{-1} v \varphi$, then $\varphi(S, v) \rightarrow\left(S, v^{\prime}\right)$ is an isomorphism of virtual semiquandles.

Every flat singular virtual knot or link has a fundamental virtual singular semiquandle, obtained by dividing the knot or link into semiarcs at flat, singular and virtual crossings; then FVSSQ $(L)$ has generators corresponding to semiarcs, and relations at the crossings as determined by crossing type, in addition to relations coming from the virtual singular semiquandle axioms.

\section{Counting invariants of flat singular virtuals}

As with finite groups, quandles, and biquandles, finite semiquandles can be used to define computable invariants of flat virtual knots and links by counting homomorphisms.

Definition 17. Let $L$ be a flat virtual link and $T$ a finite semiquandle. The semiquandle counting invariant of $L$ with respect to $T$ is the cardinality

$$
\operatorname{sc}(L, T)=|\operatorname{Hom}(\operatorname{FSQ}(L), T)|
$$

of the set of semiquandle homomorphisms $f: \operatorname{FSQ}(L) \rightarrow T$ from the fundamental semiquandle of $L$ to $T$ (that is, of maps such that $f\left(x_{y}\right)=f(x)_{f(y)}$ and $f\left(x^{y}\right)=f(x)_{f(y)}$ for all $\left.x, y \in \mathrm{FSQ}(L)\right)$. 
Remark 18. A semiquandle homomorphism $f: \operatorname{FSQ}(L) \rightarrow T$ can be pictured as a "coloring" of a diagram $D$ of $L$ by $T$, that is, as an assignment of an element of $T$ to each semiarc in $D$ such that the colors satisfy the semiquandle operation conditions at every crossing.

Example 19. The semiquandle counting invariant with respect to the semiquandle $T$ in Example 7 distinguishes the flat Kishino knot FK from the flat unknot FU, with $\operatorname{sc}(\mathrm{FK}, T)=16$ and $\operatorname{sc}(\mathrm{FU}, T)=4$. This same semiquandle also distinguishes the flat virtual knot $K$ below [Kauffman 1999] from both the unknot and the flat Kishino, with $\operatorname{sc}(K, T)=2$.

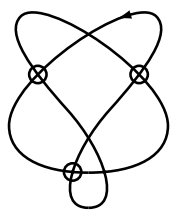

We can enhance the semiquandle counting invariant by taking note of the cardinality of the image subsemiquandles $\operatorname{Im}(f)$ for each homomorphism to obtain a multiset-valued invariant, which we can also express in a polynomial form by converting multiset elements to exponents of a dummy variable $z$ and multiplicities to coefficients. Note that specializing $z=1$ in the enhanced invariant yields the original counting invariant.

Definition 20. Let $L$ be a flat virtual link and $T$ a finite semiquandle. The enhanced semiquandle counting multiset is the multiset

$$
\operatorname{sqcm}(L, T)=\{\operatorname{Im}(f) \mid f \in \operatorname{Hom}(\operatorname{FSQ}(L), T))\},
$$

and the enhanced semiquandle polynomial is

$$
\operatorname{sqp}(L, T)=\sum_{f \in \operatorname{Hom}(\operatorname{FSQ}(L), T)} z^{|\operatorname{Im}(f)|} .
$$

For singular semiquandles, we also have counting invariants and polynomial enhanced invariants.

Definition 21. Let $L$ be a flat singular virtual link and $(T, S)$ a finite singular semiquandle. We have the singular semiquandle counting invariant

$$
\operatorname{ssc}(L,(T, S))=|\operatorname{Hom}(\operatorname{FSSQ}(L),(T, S))|,
$$

the enhanced singular semiquandle counting multiset

$$
\operatorname{ssqcm}(L,(T, S))=\{\operatorname{Im}(f) \mid f \in \operatorname{Hom}(\operatorname{FSSQ}(L),(T, S)))\},
$$

and the enhanced singular semiquandle polynomial

$$
\operatorname{ssqp}(L,(T, S))=\sum_{f \in \operatorname{Hom}(\operatorname{FSSQ}(L),(T, S))} z^{|\operatorname{Im}(f)|} .
$$


Example 22. The constant-action semiquandle $\left(X_{(132)}, O\right)$ with operator singular structure distinguishes the triple trefoil TT from the singular knot $\mathrm{SU}_{1}$ with one singular crossing and no other crossings:
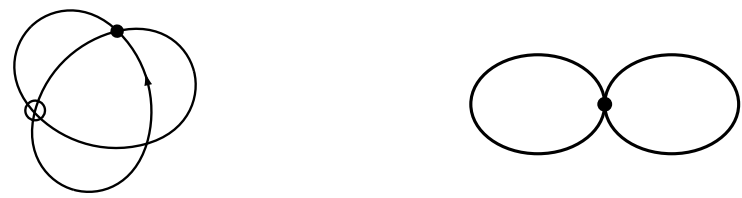

$$
\operatorname{ssqp}\left(\mathrm{TT},\left(X_{(132)}, O\right)\right)=0 \quad \operatorname{ssqp}\left(\mathrm{SU}_{1},\left(X_{(132)}, O\right)\right)=9 z^{3}
$$

Finally, we have counting invariants for flat singular virtual knots and links, defined analogously using finite virtual singular semiquandles.

Definition 23. Let $L$ be a flat singular virtual link and $(T, S, v)$ a finite virtual singular semiquandle. Then we have the virtual singular semiquandle counting invariant $\operatorname{vssc}(L,(T, S, v))=|\operatorname{Hom}(\operatorname{FVSSQ}(L),(T, S, v))|$, the enhanced virtual singular semiquandle counting multiset

$$
\operatorname{vssqcm}(L,(T, S, v))=\{\operatorname{Im}(f) \mid f \in \operatorname{Hom}(\operatorname{FVSSQ}(L),(T, S, v))\},
$$

and the enhanced virtual singular semiquandle polynomial

$$
\operatorname{vssqp}(L,(T, S, v))=\sum_{f \in \operatorname{Hom}(\operatorname{FVSSQ}(L),(T, S, v))} z^{|\operatorname{Im}(f)|} .
$$

Example 24. The flat virtual Hopf link fvH below is distinguished from the flat unlink of two components by the counting invariants with respect to the listed virtual semiquandle. Note that we can regard $T$ as a flat singular virtual semiquandle with trivial singular operations $x^{\hat{y}}=x=x_{\hat{y}}$.

$$
M_{T, S}=\left[\begin{array}{lll|lll}
1 & 3 & 1 & 1 & 3 & 1 \\
2 & 2 & 2 & 2 & 2 & 2 \\
3 & 1 & 3 & 3 & 1 & 3
\end{array}\right], \quad v=(13)
$$

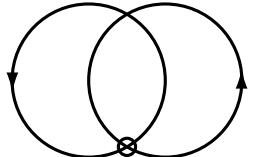

$\operatorname{vsqp}(\mathrm{fvH},(T, S, v))=q+4 z^{2}$

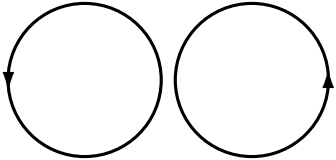

$\operatorname{vsqp}\left(U_{2},(T, S, v)\right)=q+4 z^{2}+4 z^{3}$

Remark 25. A virtual semiquandle is a virtual singular semiquandle with trivial singular structure, that is, $x^{\hat{y}}=x_{\hat{y}}=x$; a singular semiquandle is a virtual singular semiquandle with trivial virtual operation, that is, $v=\mathrm{Id}$; and a semiquandle is a virtual singular semiquandle with trivial virtual and singular structures. 


\section{Application to Vassiliev invariants}

In [Henrich 2010], one finds several degree-one Vassiliev invariants for virtual knots. One invariant, $\mathbf{S}$, takes its values in the free abelian group on the set of two-component flat virtual links. Another invariant, $\mathbf{G}$, takes its values in the free abelian group on the set of flat virtual singular knots with one singularity. It is easy to show that $\mathbf{G}$ is at least as strong as $\mathbf{S}$, but somewhat difficult to show that $\mathbf{G}$ is strictly stronger than $\mathbf{S}$. Here, we give the definitions of these invariants and provide an alternative proof that $\mathbf{G}$ is strictly stronger than $\mathbf{S}$.

Definition 26. Let $K$ be a virtual knot with diagram $\widetilde{K}$. Let $\widetilde{K}_{\text {smooth }}^{d}$ be the flat virtual link obtained by smoothing $\widetilde{K}$ at the crossing $d$ and projecting onto the associated flat virtual link. Furthermore, let $\widetilde{K}_{\text {link }}^{0}$ be the flat virtual link obtained by taking the disjoint union of the unknot with the flat projection of $\widetilde{K}$. We let the bracket $[\cdot]$ denote the associated generator of the free abelian group on the set of (two-component) flat virtual links. Then $\mathbf{S}$ is given by the following element of this free abelian group:

$$
\mathbf{S}(K)=\sum_{d} \operatorname{sign}(d)\left(\left[\widetilde{K}_{\text {smooth }}^{d}\right]-\left[\widetilde{K}_{\text {link }}^{0}\right]\right) .
$$

The sum ranges over all classical crossings in $\widetilde{K}$, and $\operatorname{sign}(d)$ is the local writhe.

Since this "smoothing" invariant has values involving flat virtual links, it is clear that semiquandles may be of use in computing $\mathbf{S}$ for pairs of virtual knots. Moreover, singular semiquandles can be put to use when computing the next invariant:

Definition 27. Let $K$ be a virtual knot with diagram $\widetilde{K}$. Let $\widetilde{K}_{\text {glue }}^{d}$ be the flat virtual singular knot obtained by gluing $\widetilde{K}$ at the crossing $d$ and projecting onto the associated flat virtual singular knot. Let $\widetilde{K}_{\text {sing }}^{0}$ be the flat virtual singular knot obtained by taking the flat projection of $\widetilde{K}$, introducing a kink via the flat Reidemeister I move, and gluing at the resulting crossing. Here, we let the bracket $[\cdot]$ denote the associated generator of the free abelian group on the set of flat virtual singular knots (with one singularity). Using this notation, we define $\mathbf{G}$ by $\mathbf{G}(K)=\sum_{d} \operatorname{sign}(d)\left(\left[\widetilde{K}_{\text {glue }}^{d}\right]-\left[\widetilde{K}_{\text {sing }}^{0}\right]\right)$. Again, the sum ranges over all classical crossings in $\widetilde{K}$ and $\operatorname{sign}(d)$ is the local writhe.

Henrich [2010] proved that both $\mathbf{S}$ and $\mathbf{G}$ are degree-one Vassiliev invariants of virtual knots, and $\mathbf{G}$ is at least as strong as $\mathbf{S}$. To show that $\mathbf{G}$ is stronger than $\mathbf{S}$, consider this pair of virtual knots in Figure 1. Call the first of these knots $K_{1}$, and the second $K_{2}$. Since the only difference between the two knots is the signs of the crossings labeled $a$ and $b$, we see that

$$
\begin{aligned}
& \mathbf{S}\left(K_{2}\right)-\mathbf{S}\left(K_{1}\right)=2\left(\left[\widetilde{K}_{\text {smooth }}^{a}\right]-\left[\widetilde{K}_{\text {smooth }}^{b}\right]\right), \quad \text { and } \\
& \mathbf{G}\left(K_{2}\right)-\mathbf{G}\left(K_{1}\right)=2\left(\left[\widetilde{K}_{\text {glue }}^{a}\right]-\left[\widetilde{K}_{\text {glue }}^{b}\right]\right) .
\end{aligned}
$$



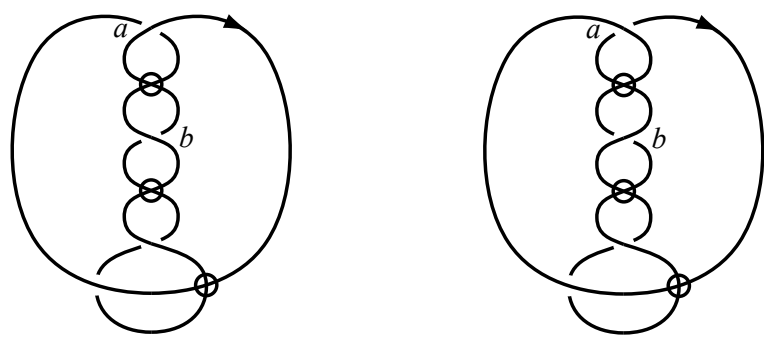

Figure 1

Now, $\widetilde{K}_{\text {smooth }}^{a}$ is the same as $\widetilde{K}_{\text {smooth }}^{b}$; they are both this flat virtual link:

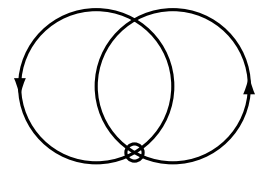

It follows that $\mathbf{S}\left(K_{1}\right)=\mathbf{S}\left(K_{2}\right)$. On the other hand, we can show, by using singular semiquandles, that $\widetilde{K}_{\text {glue }}^{a}$ and $\widetilde{K}_{\text {glue }}^{b}$, as pictured next, are distinct.
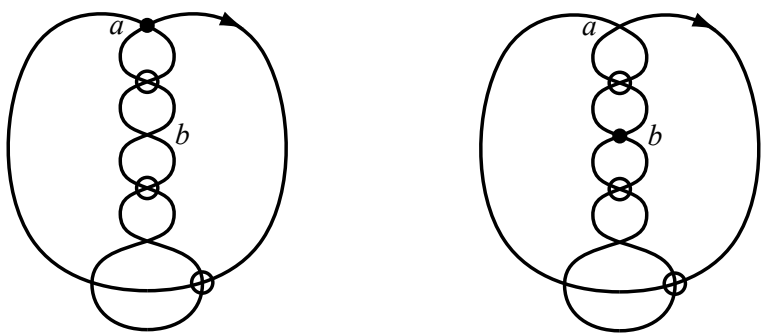

Consider the following singular semiquandle, $T$, given in terms of its matrix $M$.

$$
M=\left[\begin{array}{llll|llll}
1 & 4 & 2 & 3 & 1 & 3 & 4 & 2 \\
2 & 3 & 1 & 4 & 3 & 1 & 2 & 4 \\
4 & 1 & 3 & 2 & 2 & 4 & 3 & 1 \\
3 & 2 & 4 & 1 & 4 & 2 & 1 & 3 \\
\hline 1 & 1 & 4 & 4 & 1 & 2 & 2 & 1 \\
1 & 1 & 4 & 4 & 4 & 3 & 3 & 4 \\
2 & 2 & 3 & 3 & 4 & 3 & 3 & 4 \\
2 & 2 & 3 & 3 & 1 & 2 & 2 & 1
\end{array}\right]
$$

The enhanced singular semiquandle polynomials for $\widetilde{K}_{\text {glue }}^{a}$ and $\widetilde{K}_{\text {glue }}^{b}$ are

$$
\operatorname{ssqp}\left(\widetilde{K}_{\text {glue }}^{a}, T\right)=2 z \quad \text { and } \quad \operatorname{ssqp}\left(\widetilde{K}_{\text {glue }}^{b}, T\right)=2 z+2 z^{4} .
$$

Hence, the two flat virtual singular knots are distinct, and thus $\mathbf{G}\left(K_{1}\right) \neq \mathbf{G}\left(K_{2}\right)$. 


\section{Questions}

In this section, we collect questions for future research.

Singular semiquandles bear a certain resemblance to virtual biquandles, in which a biquandle is augmented with operations at virtual crossings. Given a biquandle $B$, the set of virtual biquandle structures on $B$ forms a group isomorphic to the automorphism group of $B$. What is the structure of the set of singular semiquandle structures on a semiquandle $X$ ?

Our algebra-agnostic approach to the computation of our various semiquandlebased invariants works well for small-cardinality semiquandles and for link diagrams with small crossing numbers. However, for links with higher crossing numbers and for larger coloring semiquandles, we will need more algebraic descriptions. We have given a few examples of classes of semiquandle structures, such as constant-action semiquandles and operator singular structures. What are some examples of group-based or module-based semiquandle and singular semiquandle structures, akin to Alexander biquandles? (Note that the only Alexander biquandles which are semiquandles are constant-action Alexander biquandles.)

Enhancement techniques for biquandle counting invariants that should extend to semiquandles include semiquandle cohomology, which is the special case of YangBaxter cohomology described in [Carter et al. 2004], and the flattened case of $S$ cohomology as described in [Ceniceros and Nelson 2009]. Similarly, we might define semiquandle polynomials and the resulting enhancements of the counting invariants, as in [Nelson 2008]. What other enhancements of semiquandle counting invariants are there?

What is the relationship, if any, between semiquandle invariants and the quaternionic biquandle invariants described in [Bartholomew and Fenn 2008]?

Our Python code for computing semiquandle-based invariants is available from the second author's website at http://www.esotericka.org.

\section{References}

[Bartholomew and Fenn 2008] A. Bartholomew and R. Fenn, "Quaternionic invariants of virtual knots and links", J. Knot Theory Ramifications 17:2 (2008), 231-251. MR 2009b:57006 Zbl 1149. 57301

[Carter et al. 2004] J. S. Carter, M. Elhamdadi, and M. Saito, "Homology theory for the set-theoretic Yang-Baxter equation and knot invariants from generalizations of quandles", Fund. Math. 184 (2004), 31-54. MR 2005k:57009 Zbl 1067.57006

[Ceniceros and Nelson 2009] J. Ceniceros and S. Nelson, "Virtual Yang-Baxter cocycle invariants", Trans. Amer. Math. Soc. 361:10 (2009), 5263-5283. MR 2010k:57021 Zbl 1182.57006

[Fenn and Rourke 1992] R. Fenn and C. Rourke, "Racks and links in codimension two", J. Knot Theory Ramifications 1:4 (1992), 343-406. MR 94e:57006 Zbl 0787.57003

[Fenn et al. 1995] R. Fenn, C. Rourke, and B. Sanderson, "Trunks and classifying spaces", Appl. Categ. Structures 3:4 (1995), 321-356. MR 96i:57023 Zbl 0853.55021 
[Henrich 2010] A. Henrich, "A sequence of degree one Vassiliev invariants for virtual knots", $J$. Knot Theory Ramifications 19:4 (2010), 461-487. MR 2646641 Zbl 05709835

[Ho and Nelson 2005] B. Ho and S. Nelson, "Matrices and finite quandles", Homology Homotopy Appl. 7:1 (2005), 197-208. MR 2006h:20094 Zbl 1102.57008

[Joyce 1982] D. Joyce, "A classifying invariant of knots, the knot quandle", J. Pure Appl. Algebra 23:1 (1982), 37-65. MR 83m:57007 Zbl 0474.57003

[Kauffman 1999] L. H. Kauffman, "Virtual knot theory", European J. Combin. 20:7 (1999), 663690. MR 2000i:57011 Zbl 0938.57006

[Kauffman and Manturov 2005] L. H. Kauffman and V. O. Manturov, "Virtual biquandles", Fund. Math. 188 (2005), 103-146. MR 2006k:57015 Zbl 1088.57006

[Kauffman and Radford 2003] L. H. Kauffman and D. Radford, "Bi-oriented quantum algebras, and a generalized Alexander polynomial for virtual links", pp. 113-140 in Diagrammatic morphisms and applications (San Francisco, 2000), edited by D. E. Radford et al., Contemp. Math. 318, Amer. Math. Soc., Providence, RI, 2003. MR 2004c:57013 Zbl 1031.57009

[Matveev 1982] S. V. Matveev, "Distributive groupoids in knot theory", Mat. Sb. (N.S.) 119 (161):1 (1982), 78-88, 160. MR 84e:57008 Zbl 0523.57006

[Nelson 2008] S. Nelson, "Generalized quandle polynomials", preprint, 2008. To appear in Can. Bull. Math. arXiv 0801.2979

[Nelson and Vo 2006] S. Nelson and J. Vo, "Matrices and finite biquandles", Homology, Homotopy Appl. 8:2 (2006), 51-73. MR 2007j:57009 Zbl 1111.57009

Received April 28, 2009.

ALLISON HENRICH

SEATTLE UNIVERSity

MATHEMATICS DEPARTMENT

901 12TH AVENUE

PO BOX 22200

SEATTLE, WA 98122

UNITED STATES

henricha@seattleu.edu

SAM NELSON

Claremont McKenna College

DEPARTMENT OF MATHEMATICAL SCIENCES

850 Columbia Ave

Claremont CA, 91711

UNITED STATES

knots@esotericka.org 


\title{
PACIFIC JOURNAL OF MATHEMATICS
}

\author{
http://www.pjmath.org \\ Founded in 1951 by \\ E. F. Beckenbach (1906-1982) and F. Wolf (1904-1989)
}

\section{EDITORS}

V. S. Varadarajan (Managing Editor)

Department of Mathematics

University of California

Los Angeles, CA 90095-1555

pacific@math.ucla.edu

Vyjayanthi Chari

Department of Mathematics

University of California

Riverside, CA 92521-0135

chari@math.ucr.edu

Robert Finn

Department of Mathematics Stanford University

Stanford, CA 94305-2125

finn@math.stanford.edu

Kefeng Liu

Department of Mathematics

University of California

Los Angeles, CA 90095-1555

liu@math.ucla.edu
Darren Long

Department of Mathematics

University of California

Santa Barbara, CA 93106-3080

long@math.ucsb.edu

Jiang-Hua Lu

Department of Mathematics

The University of Hong Kong

Pokfulam Rd., Hong Kong jhlu@maths.hku.hk

Alexander Merkurjev

Department of Mathematics

University of California

Los Angeles, CA 90095-1555

merkurev@math.ucla.edu
Sorin Popa

Department of Mathematics University of California

Los Angeles, CA 90095-1555 popa@math.ucla.edu

Jie Qing

Department of Mathematics

University of California

Santa Cruz, CA 95064

qing@cats.ucsc.edu

Jonathan Rogawski

Department of Mathematics

University of California

Los Angeles, CA 90095-1555

jonr@math.ucla.edu

\section{PRODUCTION}

pacific@math.berkeley.edu

\begin{abstract}
Silvio Levy, Scientific Editor Matthew Cargo, Senior Production Editor
\end{abstract}
ACADEMIA SINICA, TAIPEI

CALIFORNIA INST. OF TECHNOLOGY

INST. DE MATEMÁTICA PURA E APLICADA

KEIO UNIVERSITY

MATH. SCIENCES RESEARCH INSTITUTE

NEW MEXICO STATE UNIV.

OREGON STATE UNIV.

\section{SUPPORTING INSTITUTIONS}

STANFORD UNIVERSITY
UNIV. OF BRITISH COLUMBIA
UNIV. OF CALIFORNIA, BERKELEY
UNIV. OF CALIFORNIA, DAVIS
UNIV. OF CALIFORNIA, LOS ANGELES
UNIV. OF CALIFORNIA, RIVERSIDE
UNIV. OF CALIFORNIA, SAN DIEGO
UNIV. OF CALIF., SANTA BARBARA

UNIV. OF CALIF., SANTA CRUZ

UNIV. OF MONTANA

UNIV. OF OREGON

UNIV. OF SOUTHERN CALIFORNIA

UNIV. OF UTAH

UNIV. OF WASHINGTON

WASHINGTON STATE UNIVERSITY

These supporting institutions contribute to the cost of publication of this Journal, but they are not owners or publishers and have no responsibility for its contents or policies.

See inside back cover or www.pjmath.org for submission instructions.

The subscription price for 2010 is US \$420/year for the electronic version, and \$485/year for print and electronic.

Subscriptions, requests for back issues from the last three years and changes of subscribers address should be sent to Pacific Journal of Mathematics, P.O. Box 4163, Berkeley, CA 94704-0163, U.S.A. Prior back issues are obtainable from Periodicals Service Company, 11 Main Street, Germantown, NY 12526-5635. The Pacific Journal of Mathematics is indexed by Mathematical Reviews, Zentralblatt MATH, PASCAL CNRS Index, Referativnyi Zhurnal, Current Mathematical Publications and the Science Citation Index.

The Pacific Journal of Mathematics (ISSN 0030-8730) at the University of California, c/o Department of Mathematics, 969 Evans Hall, Berkeley, CA 94720-3840, is published monthly except July and August. Periodical rate postage paid at Berkeley, CA 94704, and additional mailing offices. POSTMASTER: send address changes to Pacific Journal of Mathematics, P.O. Box 4163, Berkeley, CA 94704-0163.

PJM peer review and production are managed by EditFLOW ${ }^{\mathrm{TM}}$ from Mathematical Sciences Publishers.

PUBLISHED BY PACIFIC JOURNAL OF MATHEMATICS

at the University of California, Berkeley 94720-3840

A NON-PROFIT CORPORATION

Typeset in LATEX

Copyright $(\mathrm{C} 2010$ by Pacific Journal of Mathematics 


\section{PACIFIC JOURNAL OF MATHEMATICS}

Volume $248 \quad$ No. $1 \quad$ November 2010

An existence theorem of conformal scalar-flat metrics on manifolds with boundary 1

SÉRgio de Moura Almaraz

Parasurface groups

KHALID BOU-RABEE

Expressions for Catalan Kronecker products

Andrew A. H. Brown, Stephanie Van Willigenburg and Mike

ZABROCKI

Metric properties of higher-dimensional Thompson's groups

José BURILLO and SEAN CLEARY

Solitary waves for the Hartree equation with a slowly varying potential

KIRIL DATCHEV and IVAN VENTURA

Uniquely presented finitely generated commutative monoids

PEDRO A. GARCíA-SÁNCHEZ and IgnaCio OJEDA

The unitary dual of $p$-adic $\widetilde{\operatorname{Sp}(2)}$

MARCELA HANZER and IVAN MATIĆ

A Casson-Lin type invariant for links

ERIC HARPER and NIKOLAI SAVELIEV

Semiquandles and flat virtual knots

ALLISON HENRICH and SAM NELSON

Infinitesimal rigidity of polyhedra with vertices in convex position

IVAN IZMESTIEV and JEAN-MARC SCHLENKER

Robust four-manifolds and robust embeddings

VYACHESLAV S. KRUSHKAL

On sections of genus two Lefschetz fibrations

SINEM ÇELIK ONARAN

Biharmonic hypersurfaces in Riemannian manifolds

YE-LIN OU

Singular fibers and 4-dimensional cobordism group 